\title{
MRI IN NON-TRAUMATIC VERTEBRAL COLLAPSE WITH EMPHASIS ON IN-PHASE/OPPOSED-PHASE AND DIFFUSION-WEIGHTED IMAGING SEQUENCES WITH HISTOPATHOLOGICAL CORRELATION
}

\author{
Mary Hazarika Bhuyan'1, Pradeep Kempegowda²
}

${ }^{1}$ Associate Professor, Department of Radiology, Assam Medical College, Dibrugarh.

${ }^{2}$ Registrar, Department of Radiology, Assam Medical College, Dibrugarh.

\section{ABSTRACT}

\section{BACKGROUND}

To evaluate In-Phase/Opposed-Phase and Diffusion Weighted Imaging sequences in detection and characterisation of benign versus malignant vertebral collapse and correlation of MRI findings with histopathological study.

\section{METHODS}

39 consecutive patients referred to the Department of Radiodiagnosis from various clinical departments who had symptoms related to vertebral collapse of Assam Medical College, Dibrugarh, were included in this study. They were evaluated by Siemens Magnetom Avanto 1.5T MRI scanner. The study included all OPD and indoor patients comprising of both sex of all age groups.

\section{RESULTS}

In the present study, lumbar region is more commonly involved (46.15\%) followed by thoracic region (38.46\%). L1 vertebra is the most commonly involved vertebra (7/39-17.94\%) followed by L2 (5/39 VCs - 12.82\%) and D12 (4/39- 10.25\%) vertebra. In the present study, on T1WI hypointensity was observed in all the malignant VCs and 10/17(58.82\%) cases of benign VCs, sparing of normal bone marrow signal (isointense) was observed in 7/17 (41.17\%) benign VCs. In the present study, we observed an accuracy of $92.3 \%$ in differentiating malignant from benign VCs in quantitative DWI assessment using mean ADC of $1.2 \times 10-3 \mathrm{~mm} 2 / \mathrm{s}$ as a threshold cut-off value; 2/22 malignant VCs and 1/17 benign VCs were misdiagnosed.

\section{CONCLUSION}

MRI is the most sensitive modality in the characterization of vertebral lesions and diagnosing associated complications. However, we observed good sensitivity but low specificity in distinguishing benign from malignant VCs using In-phase/Opposed-phase imaging.

\section{KEYWORDS}

MRI, Vertebral Collapse (VC), (DWI), Apparent Diffusion Coefficient (ADC).

HOW TO CITE THIS ARTICLE: Bhuyan MH, Kempegowda P. MRI in non-traumatic vertebral collapse with emphasis on inphase/opposed-phase and diffusion-weighted imaging sequences with histopathological correlation. J. Evolution Med. Dent. Sci. 2016;5(69):4958-4963, DOI: 10.14260/jemds/2016/1126

\begin{abstract}
INTRODUCTION
Invasion of the fatty bone marrow by primary malignant tumours, metastatic malignancies, benign and malignant compression fractures cause similar signal changes on routine Magnetic Resonance Imaging (MRI) studies. ${ }^{1}$ Although MRI has a high sensitivity in delineating the pathology, its specificity is low. Osteoporotic and metastatic compression fractures may be mistaken for each other in the acute phase. Oedema in the acute phase of benign fractures may replace normal bone marrow and cause hypointense signal changes in T1-weighted images and hyperintense signal changes in T2weighted images, at the same time taking contrast material. These changes are also typical for metastasis and cause confusion in diagnosis when only one lesion is present. ${ }^{[2]}$ It is quite important to determine whether a compression fracture has a malignant or a benign cause, especially in patients with a primary malignancy.
\end{abstract}

Financial or Other, Competing Interest: None.

Submission 15-12-2015, Peer Review 16-12-2015,

Acceptance 21-12-2015, Published 26-08-2016.

Corresponding Author:

Dr. Mary Hazarika Bhuyan,

C/o. Dr. R. K. Bhuyan,

$P Q 12, A M C H$,

Dibrugarh-786002.

E-mail: maryhazarikabhuyan@yahoo.com

DOI: $10.14260 /$ jemds $/ 2016 / 1126$
This is because compression fractures occur depending on osteopenia developed in these patients at a rate of one-third.[3] Therefore, in order to plan appropriate therapy according to the precise diagnosis, the patients are exposed to invasive interventions such as biopsy.[1] Diffusion-Weighted Imaging (DWI) has recently appeared as a new method of screening in characterising lesions without necessitating contrast material and in evaluating the vertebrae quantitatively. $[4,5]$

DWI provides microscopic information from water protons, which is not possible using conventional MRI. DWI measures the random (Brownian) extra-, intra- and transcellular motion of water molecules.[6] Loss of signals on DWI occurs as a result of the microscopic movements of the molecules in the diffusion-sensitivity sequences and this loss is measured by calculating the Apparent-Diffusion-Coefficient (ADC). The ADC depicts the specific diffusion capacity, microscopic structure and organization of a biological tissue. 4,7 $\mathrm{ADC}$ is a quantitative parameter calculated from DWI that combines the effects of capillary perfusion and water diffusion. ${ }^{[8]} \mathrm{ADC}$ value is calculated for each pixel of the image and is displayed as a parametric map.

By drawing regions of interests on these maps, the ADCs of different tissues can be derived.[9] Some studies have been able to differentiate acute benign compression fractures from malignant induced compression fractures according to ADC values.[2,3,10-14] Because benign vertebral fractures should contain fatty marrow and malignant processes replace normal marrow, differentiation of these two processes should be 
possible with in-phase/opposed-phase imaging. So the purpose of this study was to assess the utility of diffusionweighted MR imaging and in-phase/opposed-phase sequences for the differentiation between benign and malignant vertebral collapse and to determine the sensitivity, specificity and the accuracy in differentiating benign and malignant vertebral collapse using the optimal cut-off ADC value and signal intensity ratio in respective sequences.

\section{MATERIALS AND METHODS}

The present study was carried out in the Department of Radiodiagnosis, Assam Medical College and Hospital, Dibrugarh, for a period of one year extending from July 2014 to June 2015. Those patients who had or suspected of vertebral collapse were included. The exclusion criterion were patients with acute spinal trauma $<4$ weeks, patients with infectious cause of vertebral collapse. Complete general and physical examinations were performed. Then these patients were subjected to MRI scan. CT/USG-guided spinal biopsy were performed in doubtful cases for histopathological correlation after taking consent. Benign cases in whom imaging study were confirmatory, biopsy was not considered (Fig. 2).

The equipment was MRI (SIEMENS MAGNETOM AVANTO 1.5 TESLA whole body MRI SYSTEM). Standard surface coils and body coils were used for cervical, thoracic and lumbar spine for acquisition of images. Conventional spin echo sequences T1WI, T2WI, STIR sag, T1WI, T2WI axial, heavily $\mathrm{T} 2$ weighted (T2 haste $2 \mathrm{D}$ myelogram) and 3 plane GRE sequences were used and additional DWI with ADC sequence, sagittal in-phase and opposed-phase sequences were taken. Following administration of Gadolinium dye 3 plane T1FS sequences were taken. Collapsed vertebrae were examined for after contrast administration, signal intensity of adjacent discs, degree of marrow involvement, involvement of posterior elements, presence or absence of paraspinal mass and end-plate integrity.

In doubtful cases, tissue was subjected for Histopathological examination. The tissue was obtained from collapsed vertebra intraoperatively. CT guided spinal biopsy (Tru-Cut biopsy) was performed in cases with easy accessibility. Deeply located collapses with less probability of getting tissue specimen were not considered for biopsy. USG guided biopsy/FNAC in VCs with easy superficial accessibility of tissue.

\section{STATISTICAL ANALYSIS}

Statistical analysis was carried out with the Statistical Package for the Social Sciences for Windows 16.0 (SPSS). The results were presented as mean and standard deviation. Comparisons for a difference in mean ADC values in DWI and mean SIR in inphase/opposed-phase of the collapsed vertebrae in benign and malignant groups were conducted with an unpaired 2tailed student $\mathrm{t}$-test. $\mathrm{P}$ value $<0.05$ was considered as the statistical significance level. The optimal cut-off ADC value to separate the benign VCs from the malignant VCs was determined by Receiver Operating Characteristic (ROC) analysis. Sensitivity, specificity, positive and negative predictive values along with accuracy of these sequences were determined according to these threshold values.

The optimal cut-off ADC value of $1.2 \times 10^{-3} \mathrm{~mm}^{2}$ was used in quantitative DWI assessment in differentiating benign and malignant VCs in our study. Similar cut-off value was used by K.A.W. Abo Dewan et al (2015) [15] found "The best cut-off ADC value as calculated by the ROC curve is $>1.15$ in his study.

The optimal cut-off SIR value of 0.8 was used in in-

phase/opposed-phase imaging as a threshold to differentiate benign from the malignant VCs in our study W.K. Erly, Oh E.S. et al (2006),[16] David Disler et al (1997),[17] and Eito K et al $(2004)^{[18]}$ used similar cut-off value in their study.

\section{RESULTS}

A hospital-based cross-sectional study was carried out in the Department of Radiodiagnosis, Assam Medical College and Hospitals, Dibrugarh, from July 1st 2012 to June 30th 2013 to evaluate Diffusion Weighted Imaging (DWI) and InPhase/Opposed-Phase sequences in detection and characterisation of benign versus malignant vertebral collapse. In the present study, $61.53 \%$ of the affected population were males and $38.46 \%$ were females. We observed male and female ratio of 1.6:1. Age of presentation ranged from 18 to 74 years with a mean age of 52.97 years. More cases in the present study were noted between 60 to 70 years followed by 50 to 60 years' duration. In the present study, lumbar region is more commonly involved (46.15\%) followed by thoracic region (38.46\%). L1 vertebra is the most commonly involved vertebra (7/39-17.94\%) followed by L2 (5/39 VCs - 12.82\%) and D12 (4/39-10.25\%) vertebra.

Back pain was observed in almost all cases of VCs followed by radiating pain. Paraplegia and quadriplegia were observed in $5 / 22(22.72 \%)$ and $2 / 22(9.09 \%)$ cases of malignant VCs respectively; only 1/17 (5.88\%) benign VC (aggressive haemangioma) (Fig. 2) presented with history of progressive paraplegia. In the present study, on T1WI hypointensity was observed in all the malignant VCs and 10/17 (58.82\%) cases of benign VCs, sparing of normal bone marrow signal (isointense) was observed in $7 / 17$ (41.17\%) benign VCs.

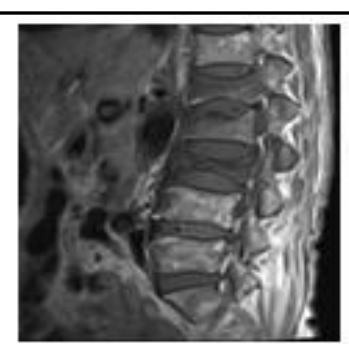

A

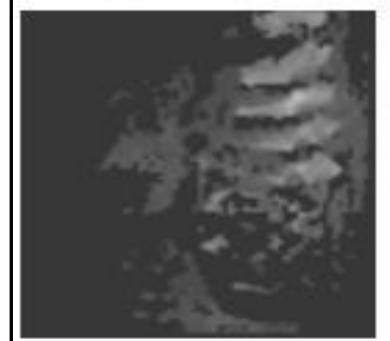

C

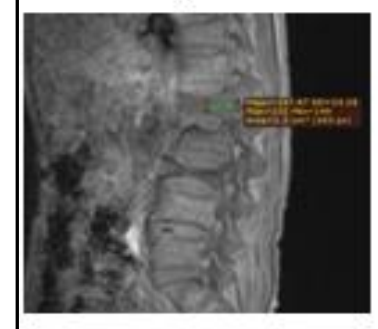

F

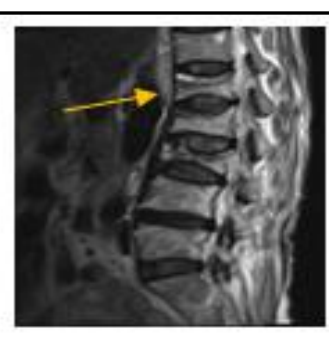

B

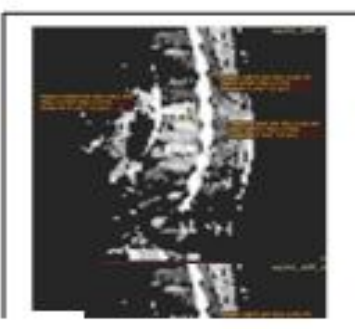

D

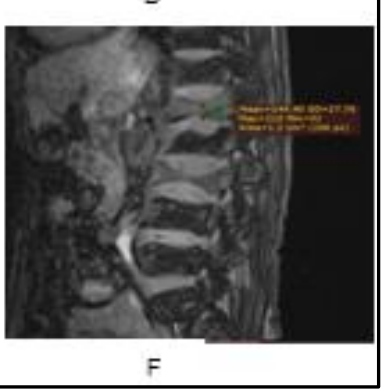

Fig. 1 
Multiple osteoporotic collapses in a 65 years female. A, B: (Sagittal T1W and T2W images) show T1 hypointense, T2 mixed iso-hypointense signal intensity and collapse of L1, L2 and L3 vertebrae, T2 cleft sign seen in L1 vertebra (Arrow). C, D: DWI show hypointense and ADC hyperintense signal in collapsed vertebrae, $\mathrm{ADC}$ value in collapsed $\mathrm{L} 1$ vertebra is 1.7 $\mathrm{x} 10^{-3} \mathrm{~mm}^{2} / \mathrm{s}$ suggestive of benign nature. E, F: (Inphase/opposed-phase images). SIR calculated by placing ROI on the collapsed L1 vertebra is 0.72 .

\begin{tabular}{|c|c|c|c|c|}
\hline \multicolumn{2}{|c|}{ Conditions } & \multirow{2}{*}{$\begin{array}{c}\begin{array}{c}\text { No. of } \\
\text { Cases }\end{array} \\
16\end{array}$} & \multicolumn{2}{|c|}{ (\%) } \\
\hline \multirow{2}{*}{ Benign } & $\begin{array}{l}\text { Osteoporotic } \\
\text { (Fig. 1) }\end{array}$ & & 41.02 & \multirow{2}{*}{43.58} \\
\hline & $\begin{array}{l}\text { Aggressive Spinal } \\
\text { Haemangioma }\end{array}$ & 1 & 2.56 & \\
\hline \multirow{4}{*}{$\begin{array}{c}\text { Primary } \\
\text { Spinal } \\
\text { Neoplasms }\end{array}$} & $\begin{array}{c}\text { Spinal } \\
\text { Lymphoma }\end{array}$ & 2 & 5.12 & \multirow{4}{*}{23.07} \\
\hline & $\begin{array}{l}\text { Multiple } \\
\text { Myeloma }\end{array}$ & 5 & 12.82 & \\
\hline & Plasmacytoma & 1 & 2.56 & \\
\hline & Chordoma & 1 & 2.56 & \\
\hline \multirow{4}{*}{$\begin{array}{c}\text { Metastasis } \\
\text { From }\end{array}$} & $\begin{array}{c}\text { Breast } \\
\text { Carcinoma }\end{array}$ & 3 & 7.69 & \multirow{5}{*}{25.64} \\
\hline & $\begin{array}{c}\text { Prostate } \\
\text { Carcinoma }\end{array}$ & 1 & 2.56 & \\
\hline & $\begin{array}{c}\text { Urinary Bladder } \\
\text { Carcinoma }\end{array}$ & 1 & 2.56 & \\
\hline & $\begin{array}{l}\text { Chronic Myeloid } \\
\text { Leukaemia }\end{array}$ & 1 & 2.56 & \\
\hline Metastasis & $\begin{array}{l}\text { Unknown } \\
\text { Primary }\end{array}$ & 4 & 10.25 & \\
\hline \multirow[b]{2}{*}{$\begin{array}{c}\text { Direct } \\
\text { Extension }\end{array}$} & PancoastTumour & 2 & 5.12 & \multirow[b]{2}{*}{7.69} \\
\hline & $\begin{array}{l}\text { Haemangioperic } \\
\text { ytoma(Fig. 3) }\end{array}$ & 1 & 2.56 & \\
\hline \multicolumn{2}{|c|}{ Total } & 39 & \multicolumn{2}{|c|}{100.00} \\
\hline \multicolumn{5}{|c|}{ Table 1: Distribution of Spinal Conditions } \\
\hline
\end{tabular}

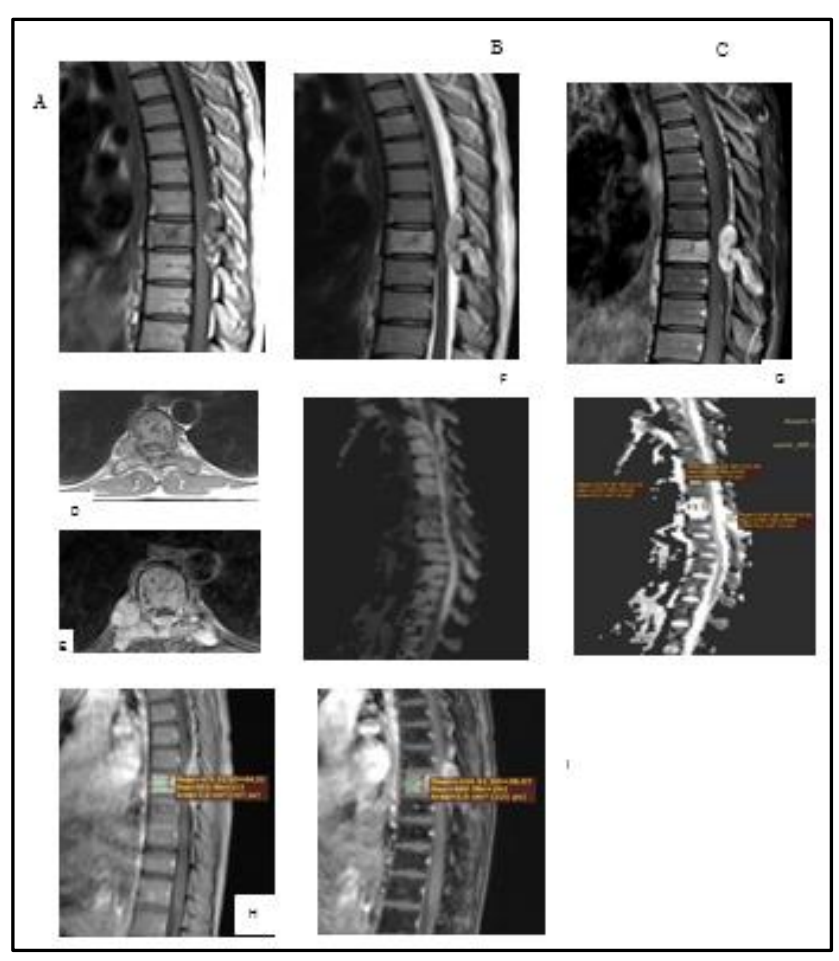

Fig. 2
Aggressive haemangioma of D8 vertebra in a 26-year-old post-partum patient presented with progressive paraplegia. A, B: (T1W and T2W images) show T1 hypointense and T2 hyperintense soft tissue mass involving D8 vertebral body and its posterior element, partial collapse of posterior element with intraspinal extension (Asterisk) leading to dorsal cord compressive myelopathy (Arrow). Multiple smaller T1 hypointensities are noted in affected vertebra (Image D) representing prominent trabeculae giving positive 'Polka Dot' sign. C, E: (Post contrast sag and axial images) show intense homogeneous enhancement of the vertebra with enhancing paraspinal and epidural soft tissue components, better seen in axial image F, G: (DWI and ADC images) show facilitated diffusion with ADC value of $2.28 \times 10^{-3} \mathrm{~mm}^{2} / \mathrm{s}$ suggesting benign nature. H, I: (In-phase/opposed-phase images). ROI cursor on the region of abnormal signal intensity, SIR is 0.70 indicating benign cause.

In the present study, on T2WI hyperintensity was observed in $20 / 22$ (90.90\%) VCs and mixed signal in $2 / 22$ (9.09\%) malignant VCs. In benign VCs, sparing of normal bone marrow signal (isointense) in $7 / 17$ (41.17\%) VCs, hypointense signal in 2/17 (11.76\%) VCs, hyperintense signal in $1 / 17$ (5.88\%) VCs and mixed signal in 7/17 (41.17\%) VCs was observed on T2WI. "T2 cleft sign" (Fig. 1B) was observed in $7 / 17(41.17 \%)$ benign VCs and none of the malignant VCs showed that sign. In the present study, DWI hyperintense and ADC hypointense restricted diffusion was observed in 20/22 (90.9\%) malignant VCs and DWI iso-hypointense and ADC hyperintense facilitated diffusion was observed in 17/17 (100\%) benign VCs and 2/22 (9.09\%) malignant VCs. In the present study, we observed an accuracy of $94.87 \%$ in differentiating malignant from benign VCs using qualitative DWI assessment. In the present study, we observed significant difference $(\mathrm{p}<0.0001)$ using unpaired student ' $\mathrm{t}$ ' test, between the mean ADC of benign and malignant VCs. In present study, $3 / 39$ (7.69\%) VCs cases were misdiagnosed using mean ADC of $1.2 \times 10^{-3} \mathrm{~mm}^{2} / \mathrm{s}$ as a threshold cut-off value in quantitative DWI assessment.

Mean ADC value below threshold cut-off were considered malignant and value above were considered as benign aetiology. In the present study, we observed an accuracy of 92.3\% in differentiating malignant from benign VCs in quantitative DWI assessment using mean ADC of $1.2 \times 10-3$ $\mathrm{mm}^{2} / \mathrm{s}$ as a threshold cut-off value; $2 / 22$ malignant VCs and $1 / 17$ benign VCs were misdiagnosed. In the present study, 9/39 (23.07\%) VCs cases were misdiagnosed using SIR of 0.8 as a threshold cut-off value in differentiating malignant from benign VCs. SIR value below threshold cut-off were considered benign and value above were considered as malignant aetiology. In the present study, we observed an accuracy of $76.92 \%$ using SIR of 0.8 as threshold in in-phase/opposedphase imaging in differentiating malignant from benign VCs; 6/17 (35.29\%) benign VCs and 3/22 (13.63\%) malignant VCs were misdiagnosed. The distribution of the spinal conditions are shown in Table 1. 


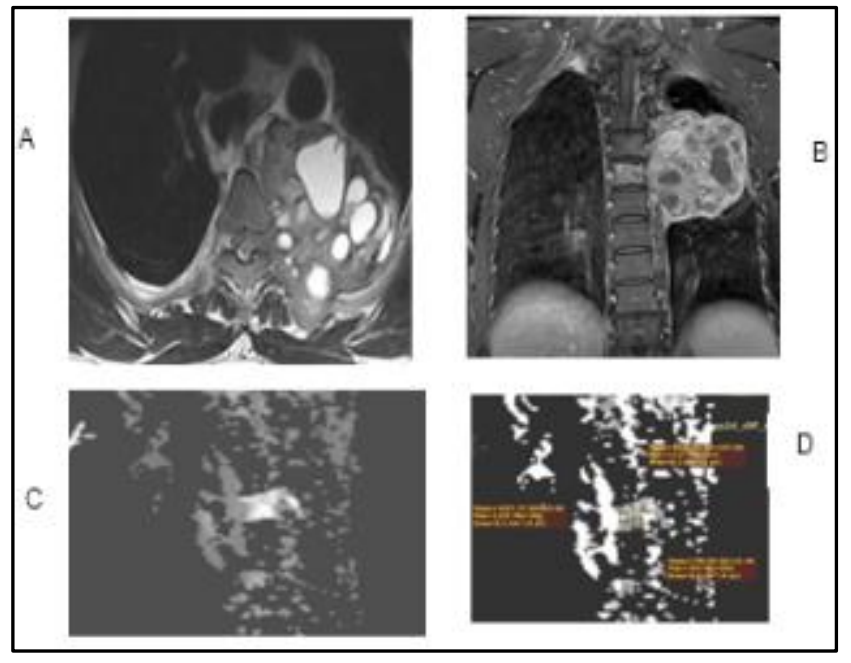

Fig. 3

Haemangiopericytoma of left $5^{\text {th }}$ rib with intraspinal extension causing D5 vertebral collapse and secondary aneurysmal bone cyst. Large mixed signal intense mass, occupying left posterior mediastinum with epicentre in left $5^{\text {th }}$ rib (Image A). Few T2 hyperintense cystic components can be seen within the lesion with multiple blood-fluid levels in axial images. Lesion extends intraspinally via left D4/5, D5/6 neural foraminae. Post contrast coronal (B) image show moderate heterogeneous enhancement. On DWI(C) affected vertebra displays high SI with low mean ADC (D) value $\left(0.936 \times 10^{-3}\right.$ $\mathrm{mm}^{2} / \mathrm{s}$ ) suggestive of malignant nature. On in-phase/opposedphase imaging affected vertebra showed SIR of 1.19.

\section{DISCUSSION}

The present study included 39 patients. Vertebral collapses were classified into two groups according to the aetiology, benign VCs and malignant VCs. The number of VCs in each group is shown in Table 1. In the present study, there were 24 (61.54\%) males and 15 (38.46\%) females with a mean age of 51.23 years. More cases in the present study were noted between 60 to 70 years followed by 50 to 60 years' duration. Our findings were similar to K.A.W. Abo Dewan et al (2015).[15] We observed 18 (46.15\%) and 15 (38.46\%) VCs in lumbar and thoracic regions respectively and 3 (7.69\%) VCs each in cervical and sacral regions. L1 vertebra was the most commonly involved vertebra $(7 / 39-17.94 \%)$ followed by L2 (5/39 VCs - 12.82\%) and D12 (4/39 - 10.25\%) vertebra.

These results matches with Turna et al (2014) ${ }^{[19]}$ studies and K.A.W. Abo Dewan et al (2015)[15] later found L1 was the most commonly fractured vertebra (23 fractures, $23.96 \%$ ) and the second most commonly fractured vertebra was D12 (20 fractures, 18.4\%). In the present study, we observed sparing of normal bone marrow signal intensity on $\mathrm{T} 1$ and $\mathrm{T} 2 \mathrm{~W}$ imaging in $41.17 \%$ cases of benign VCs respectively. These results are comparable with that reported by Jung et al (2003) $^{[20]}$ who found spared normal bone marrow signal intensity of the vertebral body was highly suggestive of acute osteoporotic compression fractures $(85 \%$ of acute osteoporotic fractures vs $19 \%$ of metastatic fractures, $\mathrm{P}$ $<0.001$ ).

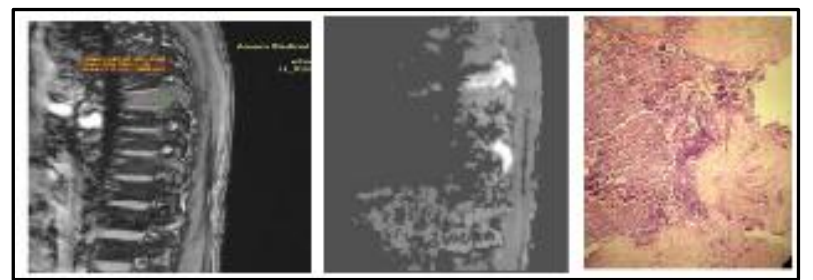

Fig. 4: Vertebral Metastasis from Squamous Cell Carcinoma of Unknown Primary. H\&E Stain of the Biopsy Tissue shows Sheets of Malignant Squamous Cells Infiltrating Normal Tissue

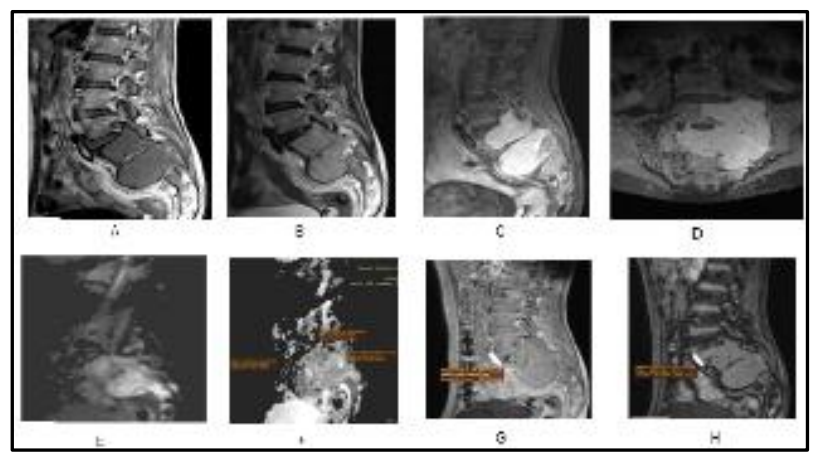

Fig. 5

Chordoma in left hemisacrum affecting S1 to S4 vertebrae with partial collapse of S1A, B: (Sagittal T1 and T2 images) show large T1 hypo and T2 hyperintense soft tissue lesion affecting the S1 to S4 vertebrae leading to partial collapse of S1 vertebra. C, D: (Post contrast sagittal and coronal) images show moderate-to-intense enhancement. E, F: (DWI and ADC map). On DWI the lesion displays high SI with low mean ADC value (1.106 x $\left.10^{-3} \mathrm{~mm}^{2} / \mathrm{s}\right)$. G, H: (In-phase/opposed-phase imaging). ROI cursor is on the region of abnormal signal intensity. SIR is 1.009 .

Transverse, linear or semilunar high signal intensity in VC on T2-weighted sagittal images called as "T2 cleft sign" (Fig. 1B) was observed in $41.17 \%$ (7/17) benign VCs and none of the malignant VC showed this sign. This finding of our study is in agreement with C.W. Yu et al (2007).[21] All cases of malignant VCs showed $\mathrm{T} 1$ hypo and $\mathrm{T} 2$ hyperintense signal was the commonly observed signal pattern except in 2 cases, which included metastatic VCs from urinary bladder and breast carcinoma respectively. This result is in agreement with G. Pozzi et al (2012) ${ }^{[22]}$ who found - In the malignant group the fractured vertebral bodies were hyperintense in $95.6 \%$ $(22 / 23)$ of lesions and hypointense in $4 \%(1 / 23)$.

W. K. Erly et al (2006)[16] who found of the 20 malignant lesions, 18 had decreased signal intensity compared with normal marrow on the T1-weighted images. On the T2weighted sequences, 4 lesions (1 each of myeloma, lymphoma, breast cancer and melanoma) were isointense to normal marrow; the remaining 16 lesions had increased signal intensity. In DWI, the reduced mobility of water in pathologic fracture is the result of tumour cell accumulation and subsequent reduction in the interstitial spaces that results in high signal intensity compared with normal bone marrow. On the other hand, the increased mobility of water attributed to an increase in the interstitial space in relation to oedema or haemorrhage[23] in benign fractures ${ }^{[2,10,24,25]}$ results in low signal intensity in benign osteoporotic and traumatic 
fractures. On this basis, DWI MRI has been suggested to be useful, particularly in the evaluation of vertebral lesions.

\section{QUALITATIVE ASSESSMENT}

In the present study, DWI hyperintense and ADC hypointense restricted diffusion was observed in 20/22 (90.9\%) malignant VCs and DWI iso-hypointense and ADC hyperintense facilitated diffusion was observed in 17/17 (100\%) benign VCs. DWI hypointensity was observed in 2/22 (9.09\%) malignant VCs. When we compared these results with histologic findings, the sensitivity and specificity of qualitative DWI study was observed to be $90.90 \%$ and $100 \%$ respectively. The positive predictive value of high signal on DWI for malignant fractures was $100 \%(20 / 20)$. If we consider malignant VCs appear hyper due to restricted diffusion and the benign VCs appear iso-hypointense on DWI, then we noticed accuracy of $94.87 \%$ in differentiating malignant VCs from benign VCs using qualitative assessment. Our findings were similar to that reported by K.A.W. Abo Dewan et al (2015),[15] Fawzy et al (2013).[26] Qualitative and Castillo et al (2000) ${ }^{[23]}$ who found qualitative assessment useful in differentiating malignant from benign cause.

\section{QUANTITATIVE STUDY}

We considered ADC value of $1.2 \times 10^{-3} \mathrm{~mm}^{2} / \mathrm{s}$ as the threshold cut-off value in differentiating benign VCs from malignant VCs; $16(94.11 \%)$ out of the 17 benign VCs and 20 (90.90\%) out of the 22 malignant VCs were correctly diagnosed. We considered ADC value of $1.2 \times 10^{-3} \mathrm{~mm}^{2} / \mathrm{s}$ as the threshold cutoff value in differentiating benign VCs from malignant VCs; 16 (94.11\%) out of the 17 benign VCs and 20 (90.90\%) out of the 22 malignant VCs were correctly diagnosed. We were able to differentiate malignant and benign lesions with sensitivity of $90.90 \%$, specificity of $94.11 \%$, positive predictive value of $95.23 \%$ and negative predictive value of $88.88 \%$ using an optimal cut-off value of $1.2 \times 10^{-3} \mathrm{~mm}^{2} / \mathrm{s}$.

We observed an accuracy of $92.30 \%$ in differentiating malignant and benign VCs using quantitative ADC values, only 3 cases were misdiagnosed. This result was in agreement with K.A.W. Abo Dewan et al (2015).[15] In our study, malignant VCs were diagnosed with high sensitivity using in-phase/opposedphase imaging. This part of result is in agreement with Donald C et al (2005)[27] and W. K. Erly et al (2006)[16] study results, later found out significant difference in the mean SIR for the benign lesions compared with the malignant lesions. If an SIR of 0.80 is chosen as a cut-off with $>0.8$ defined as malignant and $<0.8$ defined as a benign result, in-phase/opposed-phase imaging correctly identified 19 of 20 malignant lesions (Sensitivity 0.95; Specificity 0.89).

But in-phase/opposed-phase imaging showed less specificity $(64.7 \%)$ in our study with $76.92 \%$ diagnostic accuracy; $35.29 \%(6 / 17)$ cases of benign VCs failed to show significant signal suppression on opposed phase and were misdiagnosed as malignant cause. Eito $\mathrm{K}$ et al (2004) ${ }^{[18]}$ also found similar findings, he stated significant difference in SIR between benign and malignant fractures, but noted that some of the benign VCs did not suppress on the opposed-phase images. In the present study, we observed that post contrast enhancement were seen on both malignant and benign VCs; however, posterior element involvement enhancing epidural, pre- and para-vertebral masses were noted in higher percentage in malignant VCs. Table 2 shows the additional findings.

\section{SUMMARY}

Based on the findings observed on MRI scan a probable diagnosis was given, which was confirmed either by FNAC/HPE of the collapsed vertebra, which was considered as the Gold standard. Both qualitative assessment and quantitative assessment using ADC value of DWMR imaging, play a useful complementary MRI tool to characterize bone marrow lesions in order to distinguish benign from malignant VCs with high sensitivity and specificity. However, we observed good sensitivity but low specificity in distinguishing benign from malignant VCs using in-phase/opposed-phase imaging.

\section{REFERENCES}

1. Herneth AM, Dominkus M, Kurtaran A, et al. Bone metastases: new trends in diagnostic imaging. Wien Med Wochenschr Suppl 2002;113:92-4.

2. Zhou XJ, Leeds NE, McKinnon GC, et al. Characterization of benign and metastatic vertebral compression fractures with quantitative diffusion MR imaging. AJNR Am J Neuroradiol 2002;23(1):165-70.

3. Balliu E, Vilanova JC, Pelaez I, et al. Diagnostic value of apparent diffusion coefficients to differentiate benign from malignant vertebral bone marrow lesions. Eur J Radiol 2009;69(3):560-6.

4. Biffar A, Sourbron S, Dietrich O, et al. Combined diffusionweighted and dynamic contrast-enhanced imaging of patients with acute osteoporotic vertebral fractures. Eur J Radiol 2010;76(3):298-303.

5. Bammer R, Fazekas F, Augustin M, et al. Diffusion-weighted MR imaging of the spinal cord. AJNR Am J Neuroradiol 2000;21:587-91.

6. Bammer R. Basic principles of diffusion-weighted imaging. Eur J Radiol 2003;45(3):169-84.

7. Herneth AM, Guccione S, Bednarski M. Apparent diffusion coefficient: a quantitative parameter for in vivo tumor characterization. Eur J Radiol 2003;45(3):208-13.

8. Le Bihan D, Breton E, Lallemand D, et al. Separation of diffusion and perfusion in intravoxel incoherent motion MR imaging. Radiology 1988;168(2):497-505.

9. Koh DM, Collins DJ. Diffusion-weighted MRI in the body: applications and challenges in oncology. AJR Am J Roentgenol 2007;188(6):1622-35.

10. Spuentrup E, Buecker A, Adam G, et al. Diffusion-weighted MR imaging for differentiation of benign fracture edema and tumor infiltration of the vertebral body. AJR Am J Roentgenol 2001;176(2):351-8.

11. Chan JH, Peh WC, Tsui EY, et al. Acute vertebral body compression fractures: discrimination between benign and malignant causes using apparent diffusion coefficients. Br J Radiol 2002;75(891):207-14.

12. Wonglaksanapimon S, Chawalparit 0 , Khumpunnip S, et al. Vertebral body compression fracture: discriminating benign from malignant causes by diffusion-weighted MR imaging and apparent diffusion coefficient value. J Med Assoc Thai 2012;95(1):81-7.

13. Mubarak F, Akhtar W. Acute vertebral compression fracture: differentiation of malignant and benign causes by diffusion weighted magnetic resonance imaging. J Pak Med Assoc 2011;61(6):555-8. 
14. Pui MH, Mitha A, Rae WI, et al. Diffusion-weighted magnetic resonance imaging of spinal infection and malignancy. J Neuroimaging 2005;15(2):164-70.

15. Dewan KAWA, Salama AA, El habashy HMS, et al. Evaluation of benign and malignant vertebral lesions with diffusion weighted magnetic resonance imaging and apparent diffusion coefficient measurements. The Egyptian Journal of Radiology and Nuclear Medicine 2015;46(2):423-33.

16. Erly WK, Oh ES, Outwater EK. The utility of inphase/opposed-phase imaging in differentiating malignancy from acute benign compression fractures of the spine. AJNR Am J Neuroradiol 2006;27(6):1183-8.

17. Disler DG, McCauley TR, Ratner LM, et al. In-phase and outof-phase MR imaging of bone marrow: prediction of neoplasia based on the detection of coexistent fat and water. AJR 1997;169:1439-47.

18. Eito K, Waka S, Naoko N, et al. Vertebral neoplastic compression fractures: assessment by dual-phase chemical shift imaging. J Magn Reson Imaging 2004;20(6):1020-4.

19. Turna O, Aybar MD, Tuzcu G, et al. Evaluation of vertebral bone marrow with diffusion weighted MRI and ADC measurements. Istanbul Med J 2014;15:116-21.

20. Jung HS, Jee WH, McCauley TR, et al. Discrimination of metastatic from acute osteoporotic compression spinal fractures with MRI imaging. Radio Graphics 2003;23(1):179-87.
21. Yu CW, Hsu CY, Shih TTF, et al. Vertebral osteonecrosis: MR imaging findings and related changes on adjacent levels. AJNR Am J Neuroradiol 2007;28:42-7.

22. Pozzi G, Parra CG, Zerbi A, et al. Diffusion-weighted MR imaging in differentiation between osteoporotic and neoplastic vertebral fractures. Eur Spine J 2012;21(Suppl 1):123-7.

23. Castillo M, Arbelaez A, Smith JK, et al. Diffusion-MR imaging offers no advantage over routine non-contrast MR imaging in the detection of vertebral metastases. AJNR Am J Neuroradiol 2000;21(5):948-53.

24. Leeds NE, Kumar AJ, Zhou XJ, et al. Magneti cresonance imaging of benign spinal lesions stimulating metastasis: role of diffusion weighted imaging. Top Magn Reson Imaging 2000;11(4):224-34.

25. Kurunlahti M, Kerttula L, Jauhiainen J, et al. Correlation of diffusion in lumbar intervertebral disks with occlusion of lumbar arteries: a study in adult volunteers. Radiology 2001;221(3):779-86.

26. Fawzy F, Tantawy HI, Ragheb A, et al. Diagnostic value of apparent diffusion coefficient to differentiate benign from malignant vertebral bone marrow lesions. Egypt J Radiol Nucl Med 2013;44(2):265-71.

27. Zajick DC, Morrison WB, Schweitzer ME, et al. Benign and malignant processes: normal values and differentiation with chemical shift MR imaging in vertebral marrow. Radiology 2005;237(2):590-6. 